\title{
Boerhaave syndrome treated with endoscopic suturing
}

\author{
Alan Chen, MD, Raymond Kim, MD
}

Boerhaave syndrome is the spontaneous rupture of the esophagus. It may occur with severe vomiting or straining. It is associated with high morbidity and mortality. Primary surgical repair is the ideal treatment. However, in patients with multiple comorbidities who are not surgical

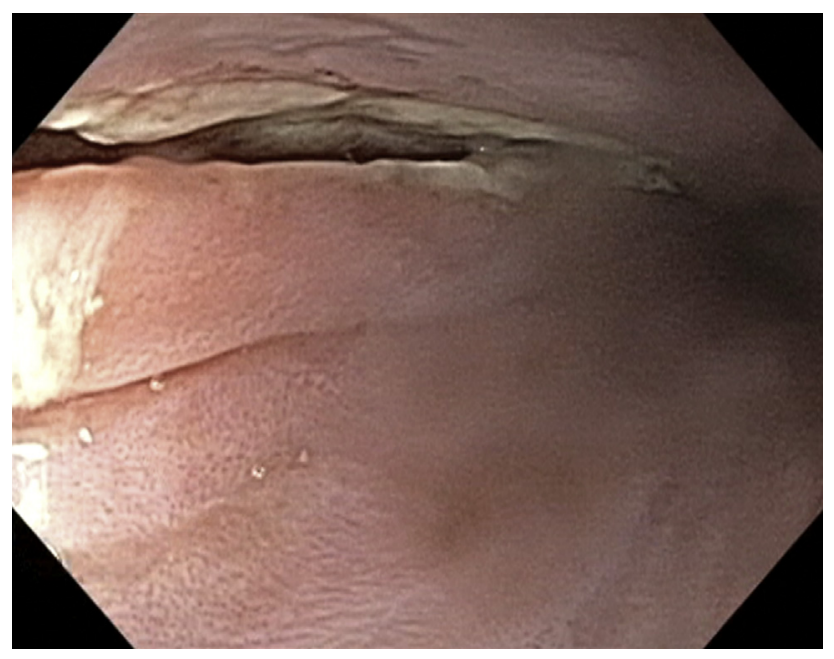

Figure 1. Esophageal perforation at the anterior wall of the distal esophagus.

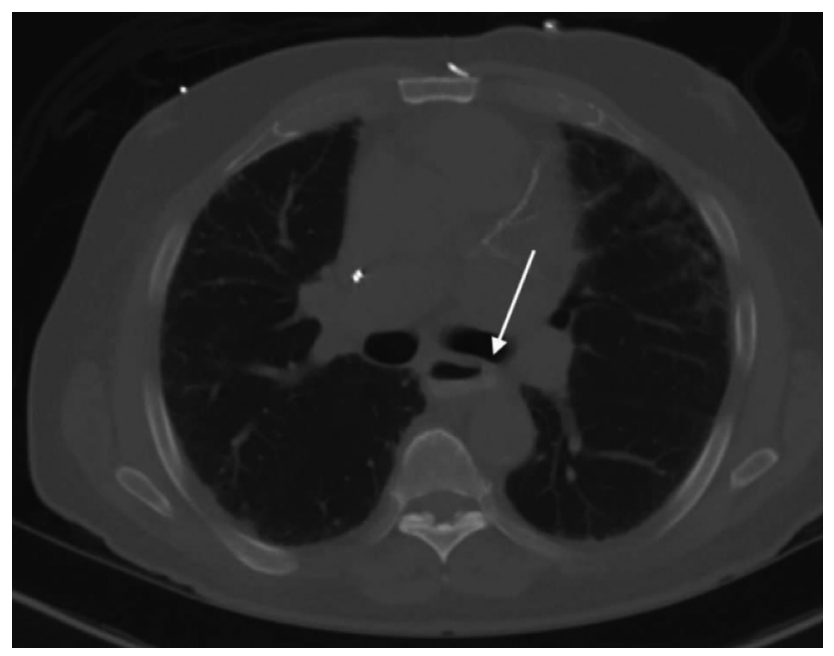

Figure 2. CT view of the thorax with oral contrast showing extraluminal extravasation of contrast material from the anterior mid to distal esophagus. candidates, treatment with esophageal stent placement can be used. Treatment with an esophageal stent frequently fails, eventually requiring surgery, and there is higher morbidity with esophageal stent placement than with primary surgical therapy. ${ }^{1}$ Endoscopic stent placement has an unclear rate of success and can be complicated by mediastinal or pleural contamination and stent migration. ${ }^{2}$ We present a case of Boerhaave syndrome treated successfully with endoscopic suturing and esophageal stent placement (Video 1, available online at www.VideoGIE.org).

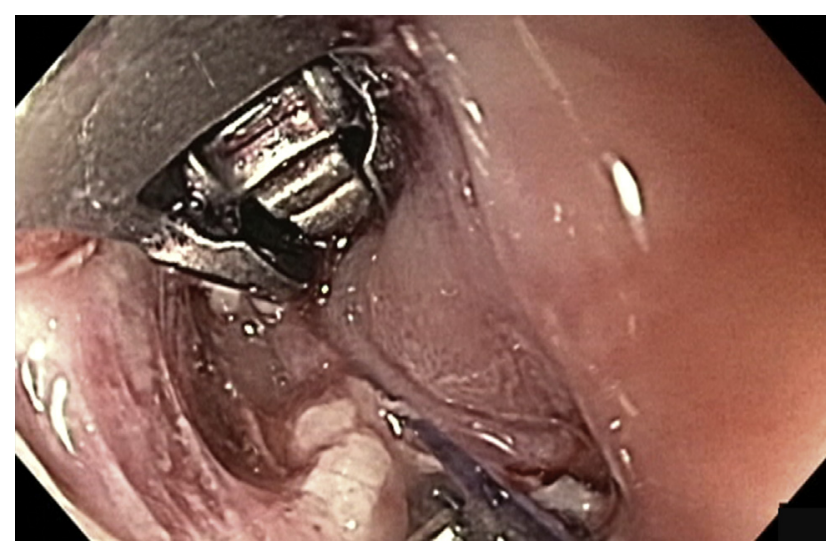

Figure 3. Endoscopic suturing device.

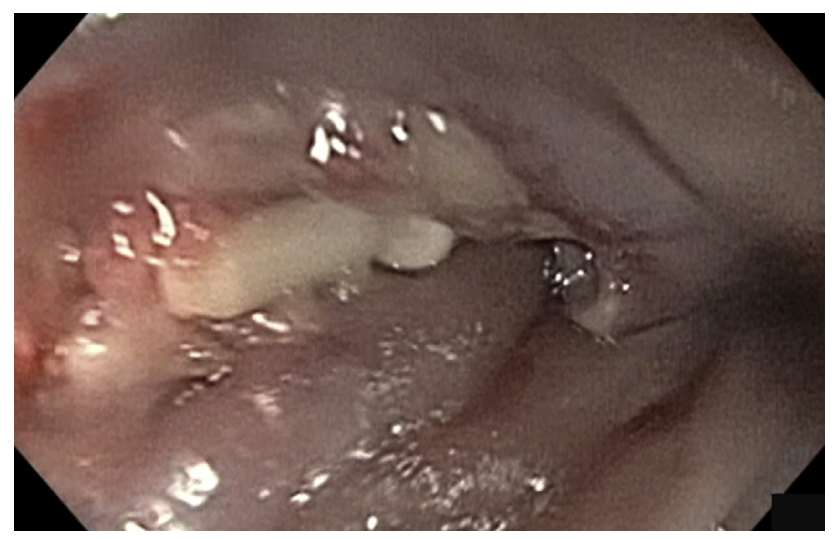

Figure 4. Suture placed in a running fashion with cinches on both ends. 


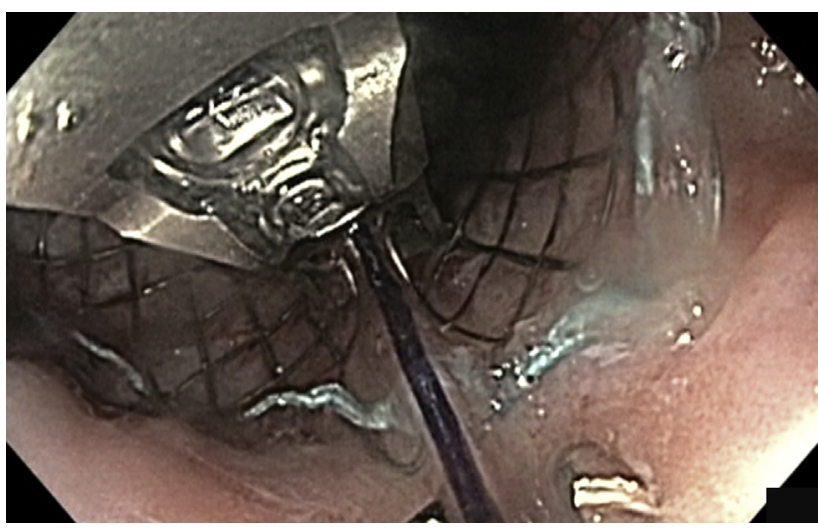

Figure 5. Esophageal stent sutured in place.

An 86-year-old man with a history of coronary artery disease, previous coronary artery bypass grafting, pulmonary hypertension, and open cholecystectomy presented to the emergency department with throat pain and substernal chest pain shortly after eating. Concern for food impaction prompted EGD, which showed an esophageal perforation at the anterior wall of the distal esophagus (Fig. 1).

Broad-spectrum antibiotics were started. CT of the thorax with oral contrast material showed extraluminal extravasation of contrast material from the anterior mid to distal esophagus consistent with a full-thickness esophageal perforation (Fig. 2). The patient was admitted to the general medicine department. A surgery consultation deemed surgery to be an extremely high risk for this patient, given his multiple comorbidities. Surgical management would involve primary repair with suturing of the perforation with reinforcement flaps, and the procedure has a favorable outcome, especially if done within 24 hours of diagnosis. ${ }^{3}$

We wanted to mimic this treatment with endoscopy. As such, the patient underwent repeated EGD, and an endoscopic suturing device with 2.0 polypropylene suture was used to suture the perforation (Fig. 3). A total of 1 suture in a running fashion was placed with cinches on both ends (Fig. 4). An 18-mm × 10.3-cm fully covered stent was then placed to cover the suture site. Endoscopic suturing with 2 interrupted sutures was performed to attach the esophageal stent to the wall of the esophagus (Fig. 5).

Three days after stent placement, a gastrograffin esophagram showed no esophageal leak. The patient's diet was advanced, and he was discharged. The esophageal stent was removed after 12 weeks. The esophageal perforation was healed (Fig. 6). The patient was asymptomatic

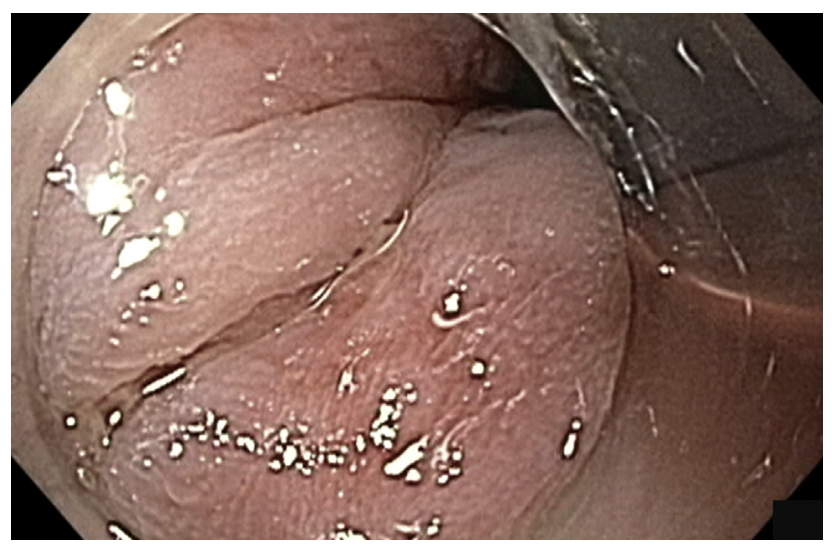

Figure 6. Healed esophageal perforation.

and tolerated a normal diet. He was doing well at the 6-month follow-up visit.

This case demonstrates the successful use of endoscopic suturing with esophageal stent placement for Boerhaave syndrome. Endoscopic suturing and stent placement can be a treatment option for patients with extensive medical comorbidities that preclude surgical management. More experience is necessary to demonstrate the efficacy of this management for Boerhaave syndrome.

\section{DISCLOSURE}

All authors disclosed no financial relationships relevant to this publication.

\section{REFERENCES}

1. Schweigert M, Beattie R, Solymosi N, et al. Endoscopic stent insertion versus primary operative management for spontaneous rupture of the esophagus (Boerhaave syndrome): an international study comparing the outcome. Am Surg 2013;79:634-40.

2. Patel SK, Ali Shera I, Raj V. Do esophageal stents have a role in Boerhaave syndrome? Case reports. Indian J Thorac Cardiovasc Surg 2018;34:78-80.

3. Romero RV, Goh K. Esophageal perforation: continuing challenge to treatment. Gastrointest Interv 2013;2:1-6.

University of Maryland, Baltimore, Maryland, USA.

Copyright (c) 2018 American Society for Gastrointestinal Endoscopy. Published by Elsevier Inc. This is an open access article under the CC BYNC-ND license (http://creativecommons.org/licenses/by-nc-nd/4.0/).

https://doi.org/10.1016/j.vgie.2018.12.005 